Service social

\title{
Les expériences de victimisation des jeunes à travers le prisme de l'intersectionnalité
}

\author{
Martin Blais, Mathieu Philibert, Félix-Antoine Bergeron et Martine Hébert
}

Volume 64, numéro 1, 2018

URI : https://id.erudit.org/iderudit/1055887ar

DOI : https://doi.org/10.7202/1055887ar

Aller au sommaire du numéro

Éditeur(s)

École de service social de l’Université Laval

ISSN

1708-1734 (numérique)

Découvrir la revue

Citer cet article

Blais, M., Philibert, M., Bergeron, F.-A. \& Hébert, M. (2018). Les expériences de victimisation des jeunes à travers le prisme de l'intersectionnalité. Service social, 64(1), 1-14. https://doi.org/10.7202/1055887ar
Résumé de l'article

Le racisme, l'hétérosexisme et le cissexisme sont des formes d'oppression qui exposent les jeunes racisés, les jeunes de la diversité sexuelle et les jeunes trans ou en questionnement de leur identité de genre à de la violence. Nous inspirant de la théorie de l'intersectionnalité, nous avons exploré les expériences de victimisation vécues par les jeunes sur une période de 12 mois en fonction de ces formes d'oppression. L'échantillon analysé est composé de 2276 personnes âgées de 14 à 23 ans recrutées à travers le Québec. Les résultats ont révélé que les personnes non hétérosexuelles, trans ou en questionnement étaient plus susceptibles de rapporter de la victimisation sur les sept indicateurs mesurés, et ce, d'autant plus si elles étaient racisées. Ces résultats révèlent la vulnérabilité de ces jeunes et illustrent la pertinence d'une approche intersectionnelle pour mieux appréhender leurs expériences de victimisation.
Tous droits réservés $₫$ Service social,
Ce document est protégé par la loi sur le droit d'auteur. L'utilisation des services d’Érudit (y compris la reproduction) est assujettie à sa politique d'utilisation que vous pouvez consulter en ligne.

https://apropos.erudit.org/fr/usagers/politique-dutilisation/ 


\title{
Les expériences de victimisation des jeunes à travers le prisme de l'intersectionnalité
}

\author{
BLAIS, Martin, Ph. D. (sociologie), sexologue, professeur \\ Université du Québec à Montréal \\ PHILIBERT, Mathieu, Ph. D. (santé publique), professeur \\ Université du Québec à Montréal \\ BERGERON, Félix-Antoine, B. A. (sexologie), candidat à la maîtrise en sexologie \\ Université du Québec à Montréal \\ HÉBERT, Martine, Ph. D. (psychologie), psychologue, professeur \\ Université du Québec à Montréal
}

\begin{abstract}
RÉSUMÉ
Le racisme, l'hétérosexisme et le cissexisme sont des formes d'oppression qui exposent les jeunes racisés, les jeunes de la diversité sexuelle et les jeunes trans ou en questionnement de leur identité de genre à de la violence. Nous inspirant de la théorie de l'intersectionnalité, nous avons exploré les expériences de victimisation vécues par les jeunes sur une période de 12 mois en fonction de ces formes d'oppression. L'échantillon analysé est composé de 2276 personnes âgées de 14 à 23 ans recrutées à travers le Québec. Les résultats ont révélé que les personnes non hétérosexuelles, trans ou en questionnement étaient plus susceptibles de rapporter de la victimisation sur les sept indicateurs mesurés, et ce, d'autant plus si elles étaient racisées. Ces résultats révèlent la vulnérabilité de ces jeunes et illustrent la pertinence d'une approche intersectionnelle pour mieux appréhender leurs expériences de victimisation.
\end{abstract}

Mots-clés : racisme, hétérosexisme, cissexisme, jeunes, intersectionnalité.

\section{Abstract}

Racism, heterosexism and cissexism are forms of oppression that expose racialized, sexually diverse and trans youths to violence. We explored youth's victimization experiences in the past 12 months according to these forms of oppression in an intersectional perspective. The sample is composed of 2,276 people aged 14 to 23 recruited throughout the province of Quebec. The findings revealed that non-heterosexual, trans or questioning participants were more likely to experience victimization, especially when they were also racialized. These results highlight the vulnerability of these youths as well as the relevance of the intersectional approach to better understand their victimization experiences.

Keywords: racism, heterosexism, cissexism, youth, intersectionality.

Remerciements : Les auteurs remercient les organismes communautaires qui ont participé au recrutement, notamment le Conseil québécois LGBT, et les jeunes qui ont participé à l'enquête. Ce travail a été financé par les Instituts de recherche en Santé du Canada (www.irsc-cihr.gc.ca) FRN : 103944. 
Les sciences sociales ont mis en lumière différentes formes d'oppression basées, notamment, sur la classe sociale, l'âge, le sexe, le genre, l'orientation sexuelle ou l'appartenance ethnique. Dans cet article, nous allons explorer l'expérience de la violence vécue par des jeunes en fonction de trois formes d'oppression - le racisme, l'hétérosexisme et le cissexisme - et leurs combinaisons. L'Organisation mondiale de la santé définit la violence comme toute "menace ou utilisation intentionnelle de la force physique ou du pouvoir contre soi-même, contre autrui ou contre un groupe ou une communauté qui entraîne ou risque fortement d'entraîner un traumatisme, un décès, des dommages psychologiques, un mauvais développement ou des privations » (Dahlberg et Krug, 2002, p. 5). La violence est désormais reconnue comme un problème de santé publique chez les jeunes (Dahlberg et Mercy, 2009 ; Mian, 2004). Ses conséquences sur les jeunes sont importantes : réduction de l'estime de soi, peur et évitement de l'école, absentéisme scolaire, symptômes de stress post-traumatique, suicidalité, consommation d'alcool et de drogues, etc. (Esbensen et Carson, 2009 ; Kowalski, Giumetti, Schroeder et Lattanner, 2014 ; Reisner, Greytak, Parsons et Ybarra, 2014).

\section{LES FORMES D'OPPRESSION ET LEURS COMBINAISONS}

Le racisme, l'hétérosexisme et le cissexisme sont des formes d'oppression qui ciblent respectivement l'origine ethnique, l'orientation sexuelle et l'identité de genre. Le racisme décrit le traitement inégal que subissent les personnes catégorisées dans des groupes distincts sur la base de caractéristiques physiques (couleur de la peau, texture des cheveux, forme des yeux, etc.) ou de leur origine ethnoculturelle (pour une discussion, voir notamment Anthias et Yuval-Davis, 1993, chapitre 1), en accordant une valeur aux caractéristiques ou origines du groupe majoritaire (en l'occurrence, en contexte québécois, aux personnes blanches de descendance européenne). L'hétérosexisme hiérarchise les individus selon leur orientation sexuelle déclarée ou présumée et il « nie, dénigre et stigmatise les comportements, les identités, les relations ou les communautés non hétérosexuels » (Herek, 1990, p. 316-317) pour accorder une plus grande valeur à l'hétérosexualité. Le cissexisme exprime l'idée que toutes les personnes désignées comme étant de sexe masculin à la naissance deviennent des hommes et que toutes les personnes désignées comme étant de sexe féminin deviennent des femmes (Bauer et al., 2009), établissant comme préférable la correspondance entre le genre et le sexe déterminé à la naissance. Le racisme, l'hétérosexisme et le cissexisme se traduisent par des préjugés dont la violence à l'égard des personnes et des groupes concernés est une des manifestations. Par exemple, une étude réalisée à Terre-Neuve a rapporté que plus de $80 \%$ des étudiantes et étudiants postsecondaires ont vécu des propos ou des comportements racistes ou en ont été témoins (Baker et Bittner, 2013). De tels incidents sont souvent minimisés ou interprétés comme des blagues par les pairs, contribuant ainsi à invisibiliser l'expérience singulière des jeunes racisés (Raby, 2004).

La victimisation hétérosexiste ou homophobe touche $22 \%$ des étudiantes et étudiants au Canada, quelle que soit leur orientation sexuelle, avec des variations de 14 à $26 \%$ selon les provinces et un risque plus élevé pour les personnes lesbiennes, gaies, bisexuelles, queer ou en questionnement (Peter, Taylor et Chamberland, 2015). Au Québec, la victimisation de nature verbale basée sur l'orientation sexuelle touche $34,7 \%$ de la population étudiante et la victimisation de nature physique, 8,5\% (Peter et al., 2015). Dans les écoles secondaires québécoises, les personnes non hétérosexuelles présentent des taux de victimisation homophobe significativement plus élevés que les personnes hétérosexuelles. Cénat, Blais, Hébert, Lavoie et Guerrier (2015) rapportent des taux de discrimination fondée sur l'orientation sexuelle sur une période de 12 mois variant de 7 à $47 \%$ chez les personnes lesbiennes, gaies ou bisexuelles de 14 à 18 ans, comparativement à $1,7 \%$ chez les personnes hétérosexuelles. Chamberland, Richard et Bernier (2013) rapportent que $69 \%$ des 
jeunes lesbiennes, gais, bisexuel.le.s ou queer (LGBQ) de 14 à 17 ans ont vécu des incidents homophobes de divers ordres, contre $35,4 \%$ des jeunes hétérosexuels. Près de la moitié des personnes d'un échantillon composé de personnel enseignant canadien disent avoir entendu des remarques homophobes tous les jours ou toutes les semaines dans leur établissement d'enseignement, une fréquence plus élevée que pour les remarques sexistes ou relatives au poids, notamment (Taylor et al., 2015).

En ce qui concerne la victimisation fondée sur l'expression de genre, elle est particulièrement élevée chez les étudiantes et étudiants trans ou en questionnement de leur identité de genre $(60 \%)$ et non exclusivement hétérosexuels ( 11 à $47 \%$ ), comparativement à des taux de 5-6 \% chez les personnes se désignant comme exclusivement hétérosexuelles et cisgenres (Blais et al., 2013). Lorsque les enseignants sont interrogés, $50 \%$ d'entre eux rapportent avoir été témoins de harcèlement, de la part d'autres étudiant.e.s, envers des garçons qui étaient considérés comme féminins et $30 \%$ envers des filles qui étaient considérées comme masculines (Taylor et al., 2015).

Dans cet article, nous allons documenter les violences vécues par des jeunes du Québec à travers le prisme du racisme, de l'hétérosexisme et du cissexisme en adoptant une perspective intersectionnelle. La théorie de l'intersectionnalité nous invite à considérer ces formes d'oppression non pas séparément, mais dans leurs combinaisons. Cette théorie postule que les personnes peuvent être exposées à plusieurs formes d'oppression simultanément et que les effets de ces formes d'oppression sur les personnes varient non seulement selon le nombre de formes d'oppression susceptibles de les affecter, mais aussi selon leurs combinaisons particulières. Par exemple, l'expérience d'une personne trans, hétérosexuelle et racisée sera différente de celle d'une personne cisgenre, racisée et gaie ou lesbienne, même si toutes deux sont affectées par deux formes d'oppression simultanément.

\section{MÉTHODE}

\section{L'enquête}

Les données utilisées pour cet article sont un sous-ensemble des données colligées auprès de deux échantillons entre 2011 et 2014 , l'un composé de jeunes recrutés dans des écoles secondaires et l'autre, de jeunes lesbiennes, gais, bisexuelles, bisexuels, trans ou en questionnement de leur orientation sexuelle ou de leur identité de genre (LGBTQ) recrutés en ligne. Le premier échantillon est composé de 8194 élèves de 14 à 18 ans de 34 écoles secondaires francophones et anglophones sélectionnées aléatoirement à travers le Québec qui ont rempli un questionnaire papier en classe. Le second échantillon est formé de 336 jeunes LGBTQ de 14 à 23 ans qui ont rempli un questionnaire en ligne. Plusieurs stratégies ont été mises en place pour recruter les jeunes LGBTQ, notamment l'affichage de publicités en ligne (Google Ads) et sur les médias sociaux (Facebook), l'envoi de courriels sur des listes de diffusion ainsi que la distribution de dépliants promotionnels dans des organismes communautaires œuvrant auprès de cette population.

Dans le cadre du présent article, seules les personnes pour lesquelles nous avons pu déterminer si elles étaient racisées ou non, cisgenres ou trans (ou en questionnement de leur identité de genre), et hétérosexuelles ou non ont été incluses. Ainsi, l'échantillon analysé dans la présente étude est composé de 2276 personnes âgées de 14 à 23 ans (moyenne $=16,0$; écart-type =1,7). Elles étaient $95 \%$ à se déclarer cisgenres et $5 \%$ à se déclarer trans ou en questionnement de leur identité de genre. Elles étaient majoritairement blanches ou d'origine caucasienne (83\%) et $17 \%$ appartenaient à un groupe racisé. En ce qui a trait à l'orientation sexuelle, $66 \%$ ont rapporté une attirance sexuelle exclusivement pour des personnes de l'autre sexe/genre et $33,9 \%$ une attirance 
non exclusivement hétérosexuelle. Une majorité de jeunes résidaient avec deux parents (57\%) au moment de l'enquête, $23 \%$ avec un parent et $13 \%$ en garde partagée. Environ une personne sur cinq était en $3^{\mathrm{e}}$ secondaire ( $22 \%$ ), $36 \%$ étaient en $4^{\mathrm{e}}$ secondaire et $28 \%$ étaient en $5^{\mathrm{e}}$ secondaire, alors que $14 \%$ étaient aux études postsecondaires. La plupart, soit $93 \%$, avaient le français comme langue le plus souvent parlée à la maison, $3 \%$, l'anglais et $4 \%$, une autre langue.

\section{Variables}

Caractéristiques individuelles ou de groupe. Nous avons classé les répondantes et les répondants selon trois caractéristiques qui pouvaient les exposer aux formes d'oppression raciste, hétérosexiste et cissexiste. L'appartenance à un groupe racisé a été déterminée à partir de la question «À quel(s) groupe(s) ethnique(s) ou culturel(s) tes parents appartiennent-ils? » Les participant.e.s ayant répondu Québécois ou Canadien, Européen de l'Ouest ou Européen de l'Est ont été classé.e.s comme personnes non racisées et les autres, comme personnes racisées (Autochtones, Premières Nations, Inuits ou Métis; Latino-Américains; Afro-Américains/Afrique noire; Asiatiques ; Caribéens/Antillais ; Afrique du Nord (Maghreb)/Moyen-Orient ; autres). L'orientation sexuelle a été déterminée à partir de la réponse à la question suivante : "Les gens sont différents dans leur façon d'être attirés par les autres. Laquelle des descriptions suivantes représente le mieux tes sentiments ? Tu es sexuellement attiré/e... ». Les personnes ayant répondu " seulement par des personnes de l'autre sexe " ont été classées comme hétérosexuelles et les autres, comme non exclusivement hétérosexuelles (y compris celles qui ont indiqué n'être attirées par personne ou être incertaines ou en questionnement). Nous avons déterminé le parcours cisgenre ou trans des jeunes à partir de l'énoncé suivant : "Quand leur sexe de naissance et leur identité de genre (sentiment d'appartenir à un sexe) ne concordent pas, certains se définissent comme une personne trans (transgenre, transsexuelle, transidentifiée). Te considères-tu comme une personne trans? » Les personnes qui ont répondu oui ou incertain/en questionnement ont été considérées comme des jeunes trans ou en questionnement de leur identité de genre et les autres, comme cisgenres.

Victimisation interpersonnelle. Six types de victimisation ont été évalués par la question suivante : "Au cours des 12 derniers mois, environ combien de fois... » Ces types étaient : I'intimidation dans les interactions en face à face, à l'école et ailleurs ("quelqu'un t'a harcelé/e (rumeurs, intimidation, menaces, etc.) à l'école ou ailleurs (excluant par voie électronique) ?») ; l'intimidation en ligne ("quelqu'un t'a harceléle (rumeurs, intimidation, menaces, etc.) par voie électronique (Facebook, Myspace, MSN, courriel, texto, etc.) ?») ; le harcèlement sexuel verbal ("as-tu été la cible de commentaires, de blagues ou de gestes à connotation sexuelle (l'autre siffle, imite une fellation, etc.) ?») ; le harcèlement sexuel physique ("une autre personne que ton chum ou ta blonde t'a touché/e, agrippé/e ou s'est frotté contre toi d'une manière sexuelle (en sachant que tu ne serais probablement pas d'accord) ?») ; la discrimination basée sur l'orientation sexuelle (" astu personnellement été traitéle de façon injuste à cause de ton orientation sexuelle ? ») ; ainsi que la discrimination basée sur l'expression de genre ("as-tu personnellement été traité/e de façon injuste parce qu'on considérait que tu étais trop féminin ou pas assez masculin/trop masculine ou pas assez féminine ? »). L'échelle de réponse allait de 0 (jamais) à 3 (6 fois ou plus). Nous avons calculé des mesures de prévalence (Jamais vs Au moins une fois) pour chacun des six types en plus d'un indicateur général indiquant le fait d'avoir vécu (1) ou non (0) au moins un des types de discrimination. 


\section{Analyses statistiques}

Nous avons analysé la prévalence des types de victimisation (variables dépendantes) en fonction des trois caractéristiques ciblées par les formes d'oppression considérées ici (variable indépendante) suivant trois approches: unitaire, multiple et intersectionnelle (Bauer, 2014). L'approche unitaire consiste à estimer les prévalences de victimisation en considérant séparément l'appartenance ou non à un groupe racisé, l'orientation sexuelle (personnes LGB ou non exclusivement hétérosexuelles, comparées aux personnes exclusivement hétérosexuelles) et l'expérience d'un parcours trans ou d'un questionnement de l'identité de genre (comparativement aux personnes présentant un parcours cisgenre au moment de l'étude). L'approche multiple consiste à comparer les prévalences de victimisation en fonction du cumul de ces caractéristiques et nous avons testé l'hypothèse d'une augmentation linéaire de la victimisation à mesure qu'augmente ce nombre (de 0 à 3). Par exemple, une personne racisée, homosexuelle et cisgenre est exposée à deux des trois formes d'oppression considérées ici (racisme et hétérosexisme) alors qu'une personne non racisée, présumée hétérosexuelle et présumée cisgenre n'est à priori exposée à aucune de ces formes d'oppression (sans considérer d'autres formes possibles non prises en compte ici). L'approche intersectionnelle utilisée ici consiste à comparer les prévalences de victimisation en fonction des huit combinaisons singulières des formes d'oppression. Pour déterminer s'il existait des différences significatives entre les groupes comparés, nous avons effectué des tests de chi carré qui permettent de déterminer si la variation des prévalences de victimisation est associée ou non aux caractéristiques considérées. Lorsque la taille des effectifs dans certaines cellules était inférieure à 5, nous avons rapporté la valeur du test exact de Fisher, qui est recommandé en raison de sa précision plus grande que celle du test du chi carré dans ces circonstances.

\section{RÉSUlTATS}

Au total, 63,1\% des répondants ont rapporté avoir vécu au moins un des types de victimisation évalués (tableau 1). Le type rapporté par le plus grand nombre de jeunes était le harcèlement sexuel verbal (35,4\%), suivi de l'intimidation, à l'école ou ailleurs $(28,4 \%)$, de la cyberintimidation (25,7\%), du harcèlement sexuel physique $(20,1 \%)$, puis de la discrimination basée sur l'expression de genre $(14,6 \%)$ ou sur l'orientation sexuelle (8,8 \%). La comparaison des groupes selon l'approche unitaire a révélé que les personnes racisées et non racisées ont subi dans des proportions égales la majorité des formes de victimisation ici étudiées, mais que les personnes racisées étaient plus nombreuses à avoir vécu du harcèlement sexuel de nature physique dans les 12 mois précédant l'enquête (26,9\%). En ce qui concerne les personnes non hétérosexuelles, elles étaient plus nombreuses que les personnes hétérosexuelles à avoir vécu les six types de victimisation considérés. Enfin, les personnes trans ou en questionnement de leur identité de genre étaient plus susceptibles que les personnes cisgenres d'avoir été victimes de cyberintimidation $(34,7 \%)$, de harcèlement sexuel verbal $(45,7 \%)$, de discrimination basée sur l'expression de genre $(52,1 \%)$ ou sur l'orientation sexuelle $(35,8 \%)$.

La comparaison des taux de victimisation selon l'approche multiple (tableau 2) a montré que le nombre de personnes rapportant des expériences de victimisation augmentait avec le cumul des caractéristiques ciblées par le racisme, l'hétérosexisme et le cissexisme, mais cette tendance linéaire n'est vraiment confirmée statistiquement que pour deux indicateurs de victimisation, soit la discrimination fondée sur l'orientation sexuelle (hétérosexiste) et celle fondée sur le genre (cissexiste). 
En nous intéressant aux expériences de violence en fonction des combinaisons des caractéristiques considérées (approche intersectionnelle), nous pouvons constater que certaines combinaisons de ces caractéristiques entraînent davantage de victimisation (tableau 3), comme le prévoit la théorie intersectionnelle. Ainsi, les personnes non racisées, hétérosexuelles et cisgenres étaient significativement moins nombreuses à rapporter des expériences de victimisation que les personnes racisées, non hétérosexuelles et trans ou en questionnement de leur identité de genre. Dans l'ensemble, les personnes qui avaient des attirances non hétérosexuelles ou encore un parcours trans (ou un questionnement de l'identité de genre) ont rapporté des prévalences plus élevées de victimisation. Les personnes exclusivement hétérosexuelles n'ont rapporté aucune expérience de cyberintimidation, de harcèlement sexuel de nature physique ou de discrimination fondée sur l'orientation sexuelle ou le genre. 
Tableau 1. Expériences de victimisation selon l'approche unitaire

\begin{tabular}{|c|c|c|c|c|c|c|c|}
\hline & $\begin{array}{l}\text { Au moins } \\
\text { une } \\
\text { expérience }\end{array}$ & $\begin{array}{l}\text { Intimidation } \\
\text { à l'école ou } \\
\text { ailleurs }\end{array}$ & $\begin{array}{l}\text { Cyber- } \\
\text { intimidation }\end{array}$ & $\begin{array}{l}\text { Harcèlement } \\
\text { sexuel verbal }\end{array}$ & $\begin{array}{l}\text { Harcèlement } \\
\text { sexuel } \\
\text { physique }\end{array}$ & $\begin{array}{l}\text { Discrimination, } \\
\text { orientation } \\
\text { sexuelle }\end{array}$ & $\begin{array}{l}\text { Discrimination, } \\
\text { genre }\end{array}$ \\
\hline \multicolumn{8}{|c|}{$\%(n)$} \\
\hline Total & $63,1(1312)$ & $28,4(588)$ & $25,7(534)$ & 35,4 (735) & $20,1(417)$ & $8,8(182)$ & $14,6(268)$ \\
\hline Personnes racisées & $64,8(225)$ & $26,7(92)$ & $26,0(80)$ & $36,5(126)$ & $26,9(93)$ & $8,4(29)$ & $15,7(48)$ \\
\hline $\begin{array}{l}\text { Personnes non } \\
\text { racisées }\end{array}$ & $62,7(1079)$ & 28,7 (493) & $23,2(447)$ & $35,2(604)$ & $18,7(322)$ & $8,8(152)$ & $14,4(218)$ \\
\hline $\begin{array}{l}\text { Personnes valeur } p \\
\text { de la différence }\end{array}$ & n.s. & n.s. & n.s. & n.s. & 0,001 & n.s. & n.s. \\
\hline $\begin{array}{l}\text { Personnes non } \\
\text { hétéro-sexuelles ou } \\
\text { en questionnement }\end{array}$ & 71,2 (1299) & $35,8(239)$ & $31,4(210)$ & $42,5(284)$ & $21,9(146)$ & 24,3 (162) & $29,0(162)$ \\
\hline $\begin{array}{l}\text { Personnes } \\
\text { hétérosexuelles }\end{array}$ & $59,0(822)$ & $24,9(346)$ & $23,1(322)$ & $32,1(446)$ & $19,1(266)$ & $1,4(20)$ & $8,2(103)$ \\
\hline $\begin{array}{l}\text { Personnes valeur } p \\
\text { de la différence }\end{array}$ & $<0,001$ & $<0,001$ & $<0,001$ & $<0,001$ & n.s. & $<0,001$ & $<0,001$ \\
\hline $\begin{array}{l}\text { Personnes trans ou } \\
\text { en questionnement }\end{array}$ & $78,9(75)$ & $36,8(35)$ & $34,7(33)$ & $45,7(43)$ & $22,1(21)$ & $35,8(34)$ & $52,1(38)$ \\
\hline Personnes cisgenres & $62,3(1237)$ & $28,0(553)$ & $25,3(501)$ & $34,9(692)$ & $20,0(396)$ & $7,5(148)$ & $13,1(230)$ \\
\hline $\begin{array}{l}\text { Personnes valeur } p \\
\text { de la différence. }\end{array}$ & 0,001 & n.s. & 0,042 & 0,036 & n.s. & $<0,001$ & $<0,001$ \\
\hline
\end{tabular}

Note : n.s. = différence non statistiquement significative. 
Tableau 2. Expériences de victimisation selon l'approche multiple

\begin{tabular}{|c|c|c|c|c|c|c|c|}
\hline & $\begin{array}{l}\text { Au moins } \\
\text { une } \\
\text { expérience }\end{array}$ & $\begin{array}{l}\text { Intimidation } \\
\text { à l'école ou } \\
\text { ailleurs }\end{array}$ & $\begin{array}{l}\text { Cyber- } \\
\text { intimidation }\end{array}$ & $\begin{array}{l}\text { Harcèlement } \\
\text { sexuel verbal }\end{array}$ & $\begin{array}{l}\text { Harcèlement } \\
\text { sexuel } \\
\text { physique }\end{array}$ & $\begin{array}{l}\text { Discrimination, } \\
\text { orientation } \\
\text { sexuelle }\end{array}$ & $\begin{array}{l}\text { Discrimination, } \\
\text { genre }\end{array}$ \\
\hline \multicolumn{8}{|c|}{$\%(n)$} \\
\hline Aucune caractéristique & $58,6(666)$ & $25,6(290)$ & $23,9(272)$ & $31,6(358)$ & 17,5 (199) & $1,2(14)$ & $7,6(78)$ \\
\hline Une caractéristique & $66,3(492)$ & $30,4(225)$ & $25,5(189)$ & $39,5(293)$ & $22,7(168)$ & $15,1(112)$ & $20,7(131)$ \\
\hline Deux caractéristiques & $76,3(129)$ & $38,1(64)$ & $36,9(62)$ & $42,5(71)$ & $25,0(42)$ & $31,7(53)$ & $36,4(51)$ \\
\hline Trois caractéristiques & $80,0(4)$ & $60,0(3)$ & $40,0(2)$ & $60,0(3)$ & $20,0(1)$ & $40,0(2)$ & $100(3)$ \\
\hline Valeur $p$, différence & $<0,001$ & 0,001 & 0,003 & $<0,001$ & 0,013 & $<0,001$ & $<0,001$ \\
\hline $\begin{array}{l}\text { Valeur } p \text {, tendance } \\
\text { linéaire }\end{array}$ & n.s. & n.s. & n.s. & n.s. & n.s. & $<0,001$ & $<0,001$ \\
\hline
\end{tabular}

Note : n.s. = différence non statistiquement significative. 
Tableau 3. Expériences de victimisation selon l'approche intersectionnelle

\begin{tabular}{|c|c|c|c|c|c|c|c|}
\hline & $\begin{array}{l}\text { Au moins } \\
\text { une } \\
\text { expérience }\end{array}$ & $\begin{array}{l}\text { Intimidation } \\
\text { à l'école ou } \\
\text { ailleurs }\end{array}$ & Cyberintimidation & $\begin{array}{l}\text { Harcèlement } \\
\text { sexuel }\end{array}$ & $\begin{array}{l}\text { Agression } \\
\text { sexuelle }\end{array}$ & $\begin{array}{l}\text { Discrimination } \\
\text { fondée sur } \\
\text { l'orientation } \\
\text { sexuelle }\end{array}$ & $\begin{array}{l}\text { Discrimination } \\
\text { fondée sur le } \\
\text { genre }\end{array}$ \\
\hline \multicolumn{8}{|c|}{$\%(n)$} \\
\hline $\mathrm{NR}-\mathrm{H}-\mathrm{C}$ & 58,6 & 25,6 & 23,9 & 31,6 & 17,5 & 1,2 & 7,6 \\
\hline \multicolumn{8}{|l|}{ Une caractéristique } \\
\hline $\mathrm{R}-\mathrm{H}-\mathrm{C}$ & 59,9 & 22,1 & 17,3 & 35,5 & 25,9 & 2,6 & 10,1 \\
\hline $\mathrm{NR}-\mathrm{H}-\mathrm{T}$ & 73,3 & 20,0 & 40,0 & 20,0 & 40,0 & & 25,0 \\
\hline NR-NH-C & 69,1 & 34,6 & 28,9 & 42,0 & 20,6 & 21,5 & 25,8 \\
\hline \multicolumn{8}{|l|}{ Deux caractéristiques } \\
\hline $\mathrm{R}-\mathrm{NH}-\mathrm{C}$ & 74,0 & 37,4 & 38,4 & 37,4 & 29,3 & 21,4 & 25,3 \\
\hline $\mathrm{R}-\mathrm{H}-\mathrm{T}$ & 50,0 & 50,0 & 0,0 & 50,0 & 0,0 & 0,0 & 0,0 \\
\hline NR-NH-T & 80,6 & 38,8 & 35,8 & 50,0 & 19,4 & 47,8 & 56,9 \\
\hline \multicolumn{8}{|l|}{ Trois caractéristiques } \\
\hline $\mathrm{R}-\mathrm{NH}-\mathrm{T}$ & 80,0 & 60,0 & 40,0 & 60,0 & 20,0 & 40,0 & 100,0 \\
\hline Valeur $p$ de la différence & $<0,001$ & $<0,001$ & $<0,001$ & $<0,001$ & 0,008 & $<0,001$ & $<0,001$ \\
\hline
\end{tabular}

Notes : $\mathrm{NR}=$ non racisé.e $; \mathrm{R}=$ racisé.e $; \mathrm{H}=$ hétérosexuel.le $; \mathrm{NH}=$ non-hétérosexuel.le $; \mathrm{C}=$ cisgenre $; \mathrm{T}=$ parcours trans ou en questionnement de l'identité de genre. 


\section{Discussion}

Dans une approche s'inspirant de la théorie de l'intersectionnalité, cet article explore l'expérience de victimisation chez des jeunes du Québec, sur une période de 12 mois, à travers trois formes d'oppression auxquelles elles et ils sont exposé.e.s en raison de leur origine ethnoculturelle, de leur orientation sexuelle ou de leur parcours trans (ou de leur questionnement sur leur identité de genre) : le racisme, l'hétérosexisme et le cissexisme. Pour ce faire, des analyses secondaires ont été réalisées sur un échantillon de 2276 jeunes recrutés au Québec, dans des écoles secondaires et en ligne. L'analyse des données selon l'approche unitaire a révélé que les personnes racisées étaient plus nombreuses à vivre du harcèlement sexuel de nature physique, mais que les proportions étaient similaires entre personnes racisées et non racisées pour les autres indicateurs de violence mesurés (intimidation à l'école ou ailleurs, cyberintimidation, harcèlement sexuel de nature verbale, discrimination fondée sur l'orientation et sur le genre, ainsi que l'expérience d'au moins l'une des six formes). Les personnes non hétérosexuelles ou en questionnement de leur orientation sexuelle étaient plus susceptibles de rapporter une expérience de violence à chacun des sept indicateurs de victimisation mesurés. Quant aux personnes trans ou en questionnement de leur identité de genre, elles étaient plus susceptibles que les personnes cisgenres d'avoir été victimes de cyberintimidation, de harcèlement sexuel verbal, de discrimination basée sur l'expression de genre ou sur l'orientation sexuelle, ainsi que de rapporter au moins l'une de ces six expériences. L'approche unitaire montre clairement une augmentation de la victimisation en fonction de l'orientation sexuelle et du parcours trans (ou du questionnement de l'identité de genre), mais ne permet pas de dégager un effet clair de l'appartenance à un groupe racisé, sauf en combinaison avec les deux autres caractéristiques. Ces observations concordent avec les travaux antérieurs qui rapportent des taux plus élevés de victimisation chez les personnes non hétérosexuelles, trans ou en questionnement (Chamberland et al., 2013 ; Peter et al., 2015), rappelant l'existence persistante de l'homophobie et de la transphobie dans les écoles et les communautés ainsi que la vulnérabilité des jeunes de la diversité sexuelle et de genre à ces violences.

Les résultats ont aussi montré que l'expérience de victimisation augmente avec le nombre de formes d'oppression auxquelles les personnes peuvent être exposées en raison de ces caractéristiques, sans que les tests statistiques puissent confirmer cette tendance pour quatre des sept indicateurs de victimisation. L'approche multiple trouve donc un soutien mitigé, probablement en raison de la faible occurrence de certains types de victimisation, mais aussi en raison de la petite taille des effectifs dans certains groupes, ce qui augmente les marges d'erreur. L'analyse d'un échantillon comportant un plus grand nombre de personnes racisées, non exclusivement hétérosexuelles et présentant un parcours trans pourrait permettre de confirmer la tendance observée.

Les analyses comparatives ont aussi révélé que l'exposition à la violence ne varie pas seulement selon le nombre de formes d'oppression, mais aussi selon leurs combinaisons spécifiques, une conclusion qui appuie la théorie intersectionnelle. Bien que les effectifs de certains sous-groupes dans la présente étude soient trop faibles pour tirer des conclusions robustes, les tableaux croisés permettent d'illustrer certaines tendances qui concordent avec la théorie de l'intersectionnalité. Ainsi, les personnes non hétérosexuelles et ayant un parcours trans (ou de questionnement de leur identité de genre) sont davantage victimes de violence, ce qui peut découler de l'hétérosexisme et du cissexisme auxquels elles sont exposées. Le poids du cissexisme apparaît clairement à travers les taux de victimisation des 
personnes trans ou en questionnement de leur identité de genre, et ce, quelles que soient les autres formes d'oppression qui les affectent. Comme plusieurs personnes des communautés LGBTQ présentent elles-mêmes des biais cissexistes, les personnes aux parcours trans ou en questionnement de leur identité de genre peuvent y être confrontées autant dans les communautés gaies, lesbiennes et bisexuelles que dans la société en général, comme le constate notamment l'étude ontarienne de Klein, Holtby, Cook et Travers (2015).

Le poids du racisme est apparu moins clairement dans l'analyse unitaire, sauf pour les formes sexualisées de victimisation que les personnes racisées rapportent dans des proportions importantes. Ces résultats font écho aux travaux sur le racisme sexuel, selon lesquels la sexualisation des personnes racisées constituerait une forme du racisme contemporain (Corneau, Després, Caruso et Idibouo, 2016). Chez les personnes racisées qui sont aussi trans (ou en questionnement de leur identité de genre) et non hétérosexuelles, l'expérience de victimisation augmente de façon importante, illustrant les effets combinés du racisme, de l'hétérosexisme et du cissexisme. Ainsi, les personnes LGBT racisées peuvent être exposées non seulement au cissexisme, tant à l'extérieur qu'à l'intérieur des communautés LGBT, mais aussi au racisme (Corneau et al., 2016 ; Logie et Rwigema, 2014 ; Nakamura, Chan et Fischer, 2013). De même, les personnes LGBT racisées sont aussi susceptibles de vivre de l'homophobie et de la transphobie au sein même de leur communauté d'origine. En effet, bien que la catégorie désignant les personnes racisées dans cette étude rassemble des groupes ethnoculturels très variés, on peut envisager que ces groupes, qui sont principalement issus de l'immigration nord-africaine, latino-américaine, caribéenne, antillaise, asiatique, afro-caribéenne ou afro-américaine, accordent tout autant ou plus d'importance aux normes hétérosexistes et cissexistes que la population générale.

Certaines combinaisons des formes d'oppression chez les personnes trans ou en questionnement de leur identité de genre n'étaient associées à aucun des types de victimisation considérés ici. Ce résultat, à priori contre-intuitif, peut s'expliquer par des facteurs conceptuels et méthodologiques. Sur le plan conceptuel, ces personnes se décrivaient comme hétérosexuelles, ce qui peut laisser penser que l'hétérosexualité protège en partie de ces types de victimisation, même lorsque les personnes sont exposées à d'autres formes d'oppression. Sur le plan méthodologique, il faut souligner que le petit nombre de personnes exposées aux trois formes d'oppression étudiées ici fait que les taux observés sont extrêmement dépendants de l'expérience de quelques personnes seulement. Si la taille de ces groupes avait été plus importante, nous aurions possiblement observé un portrait différent.

\section{Limites et implications pour la recherche}

Certaines limites importantes doivent être soulignées. La première concerne l'analyse de données secondaires qui nous oblige à composer avec des questions et des choix de réponses qui ne permettent pas de décrire adéquatement l'hétérogénéité des expériences intersectionnelles. Par exemple, les choix de réponses de l'enquête n'ont pas permis d'identifier des répondants intersexués ou non binaires. II est important que les enquêtes futures permettent l'expression des catégories à travers lesquelles les personnes se définissent elles-mêmes, y compris des catégories non binaires, pour mieux saisir la diversité des positions intersectionnelles et leurs impacts sur les personnes. Il est possible que certaines personnes aient été mal classées en conséquence. De même, parce que la formulation des questions sur le sexe ou le genre des répondants n'était pas adaptée aux personnes trans, nous n'avons pas inclus l'axe du sexisme dans nos analyses. 
Une autre limite concerne l'impossibilité de documenter certaines combinaisons et de produire des résultats robustes en raison du petit nombre de personnes exposées à plusieurs formes d'oppression. L'approche intersectionnelle exige des échantillons de très grande taille permettant de constituer des groupes d'une taille suffisante pour tester avec robustesse les effets des intersections sur l'exposition à la violence et ses conséquences. II est donc important de sur-échantillonner certains groupes qui, bien que représentant une proportion plus faible de la population générale, présentent des expériences qui agissent comme des révélateurs des formes d'oppression à l'œuvre dans la société et peuvent donc éclairer des processus dont les effets sur le bien-être et l'inclusion sont importants.

II faut aussi souligner que l'exposition à la violence varie selon les indicateurs mesurés. L'absence d'indicateurs mesurant la discrimination fondée explicitement sur l'origine ethnique a ainsi pu mener à sous-estimer les taux de victimisation chez les personnes racisées. Il est aussi probable que les personnes ayant participé à l'enquête soient aussi affectées par d'autres formes d'oppression (capacitisme, classisme, âgisme, etc.), en combinaison ou non avec les trois formes étudiées ici, ce qui peut avoir conduit cette étude à sous-estimer les formes d'oppression à l'œuvre et leur impact sur les jeunes. Les travaux quantitatifs s'inscrivant dans une approche intersectionnelle devraient donc mesurer autant des types de victimisation susceptibles d'être vécus par tout le monde que des types spécifiquement basés sur d'autres formes d'oppression, ce qui permettrait non seulement de faire progresser notre connaissance sur le vécu associé à différentes combinaisons de formes d'oppression, mais aussi de tester avec plus de rigueur la théorie intersectionnelle.

En raison de leurs limites, ces résultats demandent à être confirmés dans une nouvelle enquête, plus robuste et planifiée dans l'objectif explicite de les confirmer. Néanmoins, ils sont, dans l'ensemble, conformes à la théorie intersectionnelle et aux enquêtes empiriques sur le sujet, montrant l'exposition accrue à la violence de certains groupes aux croisements du racisme, de l'hétérosexisme et du cissexisme. Les intersections étudiées ici sont multiples et exposent à des vulnérabilités différentes, mais les personnes trans ou en questionnement de leur identité de genre ainsi que les personnes non hétérosexuelles étaient particulièrement exposées à la victimisation dans cet échantillon. Si elles appartenaient aussi à un groupe racisé, alors leur vulnérabilité à la violence était encore plus élevée. Dans une perspective de santé publique et de politiques sociales, ces résultats sont importants. Si les portraits chiffrés peuvent soutenir la mobilisation autour d'enjeux parfois invisibilisés, il est nécessaire d'enrichir les travaux quantitatifs par des approches qualitatives permettant de mettre en lumière les différents parcours des personnes concernées. 


\section{RÉFÉRENCES}

Anthias, F., et Yuval-Davis, N. (1993). Racialized Boundaries: Race, nation, gender, colour and class and the anti-racist struggle, New York, Routledge.

Baker, J., et Bittner, A. (2013). Building an inclusive society: Post-secondary youth perspectives on immigration, multiculturalism and racism in Newfoundland and Labrador, St. John's, T.-N.-L, Memorial University of Newfoundland.

Bauer, G. R. (2014). "Incorporating intersectionality theory into population health research methodology: Challenges and the potential to advance health equity », Social Science and Medicine, vol. 110, p. 10-17. https://doi.org/10.1016/j.socscimed.2014.03.022

Bauer, G. R., Hammond, R., Travers, R., Kaay, M., Hohenadel, K. M., et Boyce, M. (2009). “ “I Don't Think This Is Theoretical; This Is Our Lives: How Erasure Impacts Health Care for Transgender People ", Journal of the Association of Nurses in AIDS Care, vol. 20, n 5, p. 348-361. https://doi.org/10.1016/j.jana.2009.07.004

Blais, M., Gervais, J., Boucher, K., Hébert, M., Lavoie, F., et Équipe de recherche PAJ (2013). «Prevalence of prejudice based on sexual minority status in youth among youths in the province of Quebec (Canada) », The International Journal of Victimology, vol. 11, n² 2, p. 1-13.

Cénat, J. M., Blais, M., Hébert, M., Lavoie, F., et Guerrier, M. (2015). « Correlates of bullying in Quebec high school students: The vulnerability of sexual-minority youth », Journal of Affective Disorders, vol. 183, p. 315-321. https://doi.org/10.1016/j.jad.2015.05.011

Chamberland, L., Richard, G., et Bernier, M. (2013). « Les violences homophobes et leurs impacts sur la persévérance scolaire des adolescents au Québec », Recherches et éducations, n 8, p. 99-114.

Corneau, S., Després, L., Caruso, J., et Idibouo, C. (2016). « Les hommes noirs de Montréal qui ont des relations sexuelles avec d'autres hommes et le racisme sexuel ", Nouvelles pratiques sociales, vol. 28, n 1, p. 125-140. https://doi.org/10.7202/1039177ar

Dahlberg, L. L., et Krug, E. G. (2002). « La violence - un défi planétaire. », dans E. G. Krug, L. L. Dahlberg, J. A. Mercy, A. B. Zwi, et R. Lozano (dir.), Rapport mondial sur la violence et la santé, Genève, Suisse, Organisation mondiale de la santé, p. 1-21.

Dahlberg, L. L., et Mercy, J. A. (2009). « History of violence as a public health problem », American Medical Association Journal of Ethics, vol. 11, $\mathrm{n}^{\circ} 2$, p. 167-172. https://doi.org/10.1001/virtualmentor.2009.11.2.mhst1-0902

Esbensen, F.-A., et Carson, D. C. (2009). « Consequences of being bullied: Results from a longitudinal assessment of bullying victimization in a multisite sample of American students 》, Youth \& Society, vol. 41, n² 2, p. 209-233. https://doi.org/10.1177/0044118X09351067

Herek, G. M. (1990). «The Context of anti-gay violence: Notes on cultural and psychological 
heterosexism ", Journal of Interpersonal Violence, vol. 5, $n^{\circ} 3, \quad$ p. 316-333. https://doi.org/10.1177/088626090005003006

Klein, K., Holtby, A., Cook, K., et Travers, R. (2015). « Complicating the coming out narrative: Becoming oneself in a heterosexist and cissexist world ", Journal of Homosexuality, vol. 62, n 3 , p. 297-326. https://doi.org/10.1080/00918369.2014.970829

Kowalski, R. M., Giumetti, G. W., Schroeder, A. N., et Lattanner, M. R. (2014). "Bullying in the digital age: A critical review and meta-analysis of cyberbullying research among youth ", Psychological Bulletin, vol. 140, nº 4, p. 1073-1137. https://doi.org/10.1037/a0035618

Logie, C. H., et Rwigema, M.-J. (2014). « The normative idea of queer is a white person: Understanding perceptions of white privilege among lesbian, bisexual, and queer women of color in Toronto, Canada ", Journal of Lesbian Studies, vol. 18, $\mathrm{n}^{\circ} 2, \quad$ p. 174-191. https://doi.org/10.1080/10894160.2014.849165

Mian, M. (2004). « World report on violence and health: What it means for children and pediatricians ", The Journal of Pediatrics, vol. 145, no 1, p. 14-19. https://doi.org/10.1016/j.jpeds.2004.03.054

Nakamura, N., Chan, E., et Fischer, B. (2013). " Hard to crack: Experiences of community integration among first- and second-generation Asian MSM in Canada ", Cultural Diversity and Ethnic Minority Psychology, vol. 19, no 3, p. 248-256. https://doi.org/10.1037/a0032943

Peter, T., Taylor, C., et Chamberland, L. (2015). « A queer day in Canada: Examining Canadian high school students' experiences with school-based homophobia in two large-scale studies ", Journal of Homosexuality, vol. 62, n 2, p. 186-206. https://doi.org/10.1080/00918369.2014.969057

Raby, R. (2004). « "There's no racism at my school, it's just joking around”: Ramifications for anti-racist education ", Race Ethnicity and Education, vol. 7, $\mathrm{n}^{\circ} 4, \quad$ p. 367-383. https://doi.org/10.1080/1361332042000303388

Reisner, S. L., Greytak, E. A., Parsons, J. T., et Ybarra, M. L. (2014). " Gender minority social stress in adolescence: Disparities in adolescent bullying and substance use by gender identity », The Journal of Sex Research, vol. 52, p. 1-14. https://doi.org/10.1080/00224499.2014.886321

Taylor, C., Peter, T., Campbell, C., Meyer, E., Ristock, J., et Short, D. (2015). «The Every Teacher Project on LGBTQ-inclusive education in Canada's K-12 schools: Final report », Winnipeg, MB, Manitoba Teacher's Society. 